\title{
Improving Resident Satisfaction with Post-Hospitalization Follow-up at Mayo Clinic Florida
}

\author{
Megan Melody ${ }^{1}$, Samantha Espinosa ${ }^{1}$, Karl Mareth ${ }^{1}$, Lindsay Gardner ${ }^{1}$, Alexander \\ Heckman $^{1}$, Annamaria Mechtler ${ }^{1}$, SeQuoya Killebrew ${ }^{1}$, Matthew Dudgeon ${ }^{1}$, Richard \\ White $^{1}$, and Leigh Speicher ${ }^{1}$ \\ ${ }^{1}$ Mayo Clinic Florida
}

May 11, 2020

\begin{abstract}
Rationale, aims, and objectives: Post-hospitalization follow-up within 30 days following discharge has been shown to positively impact time to readmission, healthcare costs, and patient self-reported adherence and satisfaction. We aimed to improve resident satisfaction with the process of establishing post-hospitalization, primary care provider (PCP) follow-up. Methods: In this quality improvement study we surveyed all internal medicine residents at our institution regarding their satisfaction with the process of establishing PCP follow-up at the time of hospital discharge. A streamlined process was developed and two subsequent interventions were enacted; a dedicated teaching session and distribution of pocket cards outlining the process. Residents were then surveyed following each intervention to assess for impact on overall satisfaction and burden of work. Results: Initially, $77.3 \%$ of residents were not satisfied with the process of establishing post-hospitalization PCP follow-up. Following the first intervention there was a trend towards increasing satisfaction rates by $16.7 \%(\mathrm{p}=0.20)$, and after the second intervention there was a statistically significant increase in satisfaction rates from baseline by $44 \%(\mathrm{p}=0.007)$. There was also a reduction in the feeling of workload burden associated with establishing PCP follow-up from $32 \%$ to $25 \%$, and over $67 \%$ of participants either agreed or strongly agreed that the workload was not too burdensome. Conclusion: This quality improvement initiative established that resident physicians at our institution previously found the process of establishing PCP follow-up at the time of patient discharge both confusing and burdensome. Through the implementation of our interventions we were able to achieve our aims of improving resident satisfaction.
\end{abstract}

\section{Improving Resident Satisfaction with Post-Hospitalization Follow-up at Mayo Clinic Florida} Introduction:

The transition from inpatient medical care to the outpatient setting is a high risk time for patients and relies heavily on communication and coordination of medical care across multiple settings ${ }^{1-4}$. Studies have shown that patients who receive follow-up medical care within the first 30-days following hospital discharge have decreased readmission rates, decreased health care costs, and improved medical adherence and satisfaction 4-6. However, literature shows that communication between hospital physicians and primary care providers (PCP) occurs infrequently (only 3\%-20\%) and only 56\% of PCPs were satisfied with communication with hospitalists ${ }^{2,3,7-12}$. Improvements in provider satisfaction have been seen with the utilization of pre-formatted electronic communication, computer generated discharge summaries, and standardized processes to improve

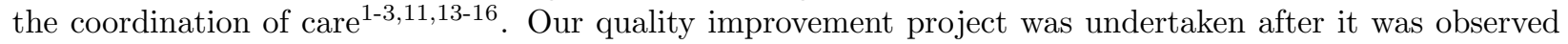
that there was no standardized process for scheduling a patient's PCP follow-up appointment at the time of hospital discharge, causing increase burden of workload for the resident and leading to dissatisfaction. Our aim was to improve resident satisfaction rating of the post-hospitalization PCP follow-up process by $10 \%$ over the course of nine months, without increasing resident perception of workload or burden. 


\section{Methods:}

Our team conducted a prospective quality improvement project with the specific intention to evaluate and improve a specific practice or program, which per institutional guidance was exempt from Institutional Review Board review. Utilizing an online survey generator, Survey Monkey®, we sent a link via email for an anonymous survey to 49 internal medicine residents. The survey consisted of one question identifying the resident's year of training, two Likert-scale questions, and four open-answer questions (Figure 1) to assess baseline resident satisfaction and workload burden associated with establishing PCP follow-up at the time of patient discharge from the hospital. Following the results of the first survey, our team identified factors leading to dissatisfaction among residents in the discharge planning process (Figure 2) and created a flow diagram outlining the current process for establishing PCP follow-up at the time of discharge (Figure 3). Our team then worked within the confines of our current electronic medical record (EMR) system to create a standardized, streamlined process for establishing patient follow-up at the time of discharge. This process included step-by-step instructions to locate PCP information in the chart; provide patient with discharge instructions; order follow-up appointments, labs, and tests; and communicate with PCPs both inside and outside of our institution's healthcare network. Our first intervention, an instructional conference held at resident noon conference, outlined this new streamlined process. Six weeks after the educational session, a second, four-question survey was sent to 49 residents, using the same method as previously outlined. This survey again contained one question identifying the resident's year of training, the same two Likert-scale questions, and one multiple choice question to assess whether the resident had attended the teaching session. For our second quality improvement intervention, pocket cards (Figure 4) were distributed to residents via email and hung on the wall of all inpatient resident work stations. Following the second intervention, a third, five-question, anonymous survey was sent to all internal medicine residents. This survey again contained one question identifying the resident's year of training, the same two Likert-scale questions, one multiple choice question to assess whether the resident had attended the initial teaching session, and an additional multiple choice question to assess whether the resident had referenced the instructional pocket cards. Data from all surveys were compiled using Survey Monkeyß software and exported to excel for statistical analysis. Data analysis was completed using the Mann Whitney U test.

Results:

The initial survey had a $45 \%$ resident response rate (22 of 49). Of these residents, five (22.7\%) were satisfied with the post-hospitalization primary care follow-up process, 11 (50\%) were neither satisfied nor dissatisfied, and six $(27.3 \%)$ were dissatisfied (Figure 5).

There was a large variation in responses to the free text question asking residents to identify their role in establishing post-hospitalization PCP follow-up. Some residents identified their primary role as communicating with the patients' PCP, with responses such as "letter to PCP", "call outside PCP", "route discharge summary to their (patient's) PCP's fax", or "messaging the PCP to let them know that their patient was hospitalized". Other residents identified their primary role as ordering, or placing a request for, follow-up appointments with the following responses: "placing an order on discharge", "usually request follow-up but not involved in scheduling", "putting in the order", and "placing an order and hoping it happens". Lastly, some residents stated that their role in post-hospitalization follow-up was to advise the patient to contact their PCP office to independently make follow-up appointments with responses including: "collect the PCP information and put it in the patient discharge instructions", "making sure the patient knows to ask outside PCP for appointment", "advise the patient to see their own PCP within the week", and "let the patient schedule their own follow-up". In response to the question "What aspects of the post-hospitalization primary care follow up process work well?" residents primarily identified one component, with nine out of the 21 free text responses stating that establishing patient follow-up was much easier within the Mayo Clinic Health Care Network. Another six out of the 21 residents responded with variations of "I don't know" or "I am not sure".

Twenty residents provided free text responses to the question "What aspects of the post-hospitalization primary care follow-up process don't work well"? Residents identified difficulty in communicating with PCPs 
(especially when outside the Mayo network), incomplete documentation or difficulty finding documentation of patients' PCP information in the EMR, and uncertainty about whether follow-up appointments actually occur. Of the 17 residents who answered the question "What do you think would improve how arrangements are made for PCP follow-up?", six residents suggested increased administrative support, five residents described standardized order sets and protocols, four residents recommended improved means for communication with PCP, one resident asked for clearer documentation of patient's PCP contact information, and one resident was unsure.

Following our first intervention, 34 out of 49 residents (69.4\%) responded to the survey and, although not statistically significant, there was an improvement when compared to the initial survey by $16.7 \%(\mathrm{p}=0.20)$. Two residents (6.1\%) reported being very satisfied with the process, 11 (33.3\%) satisfied, $14(42.4 \%)$ neither satisfied nor dissatisfied, and $6(18.2 \%)$ dissatisfied (Figure 5). Following our second intervention, 12 of the 49 residents $(24 \%)$ responded to the third survey. There was a statistically significant increase in satisfaction rates from baseline by $44 \%(\mathrm{p}=0.007)$. Two out of twelve $(16.7 \%)$ responding residents reported being very satisfied with the post-hospitalization primary care follow-up process, six (50\%) satisfied, four (33.3\%) neither satisfied nor dissatisfied, and zero residents reported being dissatisfied or very dissatisfied (Figure 5).

Prior to any intervention, the baseline survey showed only one out of $22(4.5 \%)$ responding residents strongly agreed that the time and workload associated with establishing primary care follow-up post hospitalization was not too burdensome. Seven out of $22(31.8 \%)$ agreed, seven $(31.8 \%)$ neither disagreed or agreed, and seven $(31.8 \%)$ disagreed (Figure 6). Following the first intervention there was no increase in perceived workload burden $(\mathrm{p}=0.58)$. Three of $34(8.8 \%)$ residents strongly agreed that the workload was not too burdensome, $14(41.2 \%)$ agreed, six (17.6\%) neither agreed nor disagreed, 10 (29.4\%) disagreed, and one (2.9\%) strongly disagreed (Figure 6). After the second intervention, there again was no increase in perceived workload burden from baseline ( $\mathrm{p}=0.19)$. Two out of 12 residents (16.7\%) strongly agreed, six (50\%) agreed, one (8.3\%) neither agreed nor disagreed, three (25\%) disagreed, and zero residents strongly disagreed (Figure 6).

\section{Discussion:}

Timely post-hospitalization follow-up has been shown to significantly reduce hospital readmission rates ${ }^{1,4-6}$. Transition of patient care from the inpatient to the outpatient setting can lead to missed test results, medical errors, and both patient and provider dissatisfaction in the discharge process ${ }^{7-9,17-19}$. Our initial baseline resident survey identified areas of resident dissatisfaction with the discharge planning process to include: confusion about the residents' role, difficulty in communication, and lack of a standardized process for establishing PCP follow-up. We conducted a prospective quality improvement initiative to improve resident physician satisfaction with establishing post-hospitalization PCP follow-up.

In an effort to elicit unbiased responses all survey responses were anonymous without the ability to retrospectively link resident responses with identifying information. For the first two surveys, our team was able to achieve acceptable resident response rates, with $45 \%$ and $65 \%$ of all internal medicine residents responding, respectively. Although there was a statistically significant increase in resident satisfaction rates from the initial survey to the last survey, the response rate to the last survey was significantly lower than the prior two surveys with only $24 \%$ of residents responding. Response rates to the final survey may be attributed to alert or email fatigue, as this was a longitudinal process requiring resident participation throughout the length of nearly an entire academic year ${ }^{20-22}$.

Our team was able to utilize our current hospital and clinic infrastructure and work within the technological confines of our current EMR to develop a standardized and streamlined process for establishing post hospitalization PCP follow-up. This process also included pathways to augment follow-up with both PCPs within our clinic system and PCPs at outside institutions. Through the use of two simple interventions focused on provider education, we were able to implement this new method at no additional cost to our institution. However, the ongoing use of this standardized process will require continued efforts from our quality improvement team or additional support from our institution. As current trainees graduate and new trainees enter into the residency program each year, there will need to be annual educational sessions to 
inform new residents of this process. Additionally, each resident will need to be provided the instructional pockets cards, whether via e-mail or in print, each year and the pocket cards at resident work-stations will need to be maintained.

Although this initiative was able to show an improvement in resident physician satisfaction, the intervention and analysis were limited to a small subset of resident physicians within one department and at only one academic medical center. This project did not evaluate if senior physicians and faculty or providers in other specialties encounter the same baseline dissatisfaction with establishing post-hospitalization follow-up. In addition, the trainees involved in this quality improvement project have time each day designated for education, making our interventions of provider education easier to implement. It is unclear whether this initiative, if expanded hospital wide, would have as high a yield of participation and therefore, as significant an impact on overall provider satisfaction. However, the consolidated process that we identified relied highly on tools already built into the EMR, so it is likely that this process could be more easily generalized to other services and departments at our institution or others hospitals.

Conclusion: This quality improvement initiative identified factors contributing to resident dissatisfaction with the process of establishing post-hospitalization PCP follow-up. The implementation of a standard and streamlined process resulted in a statistically significant increase in resident satisfaction with establishing PCP follow-up at the time of hospital discharge without an increase in perceived workload burden. Further efforts are required to continue the utilization of this process and to assess if this same process can increase provider satisfaction across all levels of training and specialties.

\section{References:}

1. Herzig SJ, Schnipper JL, Doctoroff L, et al. Physician Perspectives on Factors Contributing to Readmissions and Potential Prevention Strategies: A Multicenter Survey. J Gen Intern Med.2016;31(11):1287-1293.

2. Holleck JL, Gunderson CG, Antony SM, et al. The "Hand-in" Project: Jump-starting Communication Between Inpatient and Outpatient Providers.South Med J. 2017;110(11):694-698.

3. Jones $\mathrm{CD}, \mathrm{Vu} \mathrm{MB}$, O'Donnell CM, et al. A failure to communicate: a qualitative exploration of care coordination between hospitalists and primary care providers around patient hospitalizations. J Gen Intern Med. 2015;30(4):417-424.

4. Misky GJ, Wald HL, Coleman EA. Post-hospitalization transitions: Examining the effects of timing of primary care provider follow-up.J Hosp Med. 2010;5(7):392-397.

5. Lee KK, Yang J, Hernandez AF, Steimle AE, Go AS. Post-discharge Follow-up Characteristics Associated With 30-Day Readmission After Heart Failure Hospitalization. Med Care. 2016;54(4):365-372.

6. Odeh M, Scullin C, Fleming G, Scott MG, Horne R, McElnay JC. Ensuring continuity of patient care across the healthcare interface: Telephone follow-up post-hospitalization. Br J Clin Pharmacol.2019;85(3):616-625.

7. Davis MM, Devoe M, Kansagara D, Nicolaidis C, Englander H. "Did I do as best as the system would let me?" Healthcare professional views on hospital to home care transitions. J Gen Intern Med.2012;27(12):16491656 .

8. Graumlich JF, Novotny NL, Nace GS, Aldag JC. Patient and physician perceptions after software-assisted hospital discharge: cluster randomized trial. J Hosp Med. 2009;4(6):356-363.

9. Graumlich JF, Novotny NL, Stephen Nace G, et al. Patient readmissions, emergency visits, and adverse events after software-assisted discharge from hospital: cluster randomized trial.J Hosp Med. 2009;4(7):E11-19.

10. Kripalani S, Jackson AT, Schnipper JL, Coleman EA. Promoting effective transitions of care at hospital discharge: a review of key issues for hospitalists. J Hosp Med. 2007;2(5):314-323.

11. Kripalani S, LeFevre F, Phillips CO, Williams MV, Basaviah P, Baker DW. Deficits in communication and information transfer between hospital-based and primary care physicians: implications for patient safety 
and continuity of care. JAMA. 2007;297(8):831-841.

12. Nguyen OK, Kruger J, Greysen SR, Lyndon A, Goldman LE. Understanding how to improve collaboration between hospitals and primary care in postdischarge care transitions: a qualitative study of primary care leaders' perspectives. J Hosp Med. 2014;9(11):700-706.

13. Burke RE, Kripalani S, Vasilevskis EE, Schnipper JL. Moving beyond readmission penalties: creating an ideal process to improve transitional care. J Hosp Med. 2013;8(2):102-109.

14. Lang E, Afilalo M, Vandal AC, et al. Impact of an electronic link between the emergency department and family physicians: a randomized controlled trial. CMAJ. 2006;174(3):313-318.

15. Motamedi SM, Posadas-Calleja J, Straus S, et al. The efficacy of computer-enabled discharge communication interventions: a systematic review. BMJ Qual Saf. 2011;20(5):403-415.

16. Samal L, Dykes PC, Greenberg J, et al. The current capabilities of health information technology to support care transitions. AMIA Annu Symp Proc. 2013;2013:1231.

17. Callen J, Georgiou A, Li J, Westbrook JI. The safety implications of missed test results for hospitalised patients: a systematic review.BMJ Qual Saf. 2011;20(2):194-199.

18. Leppin AL, Gionfriddo MR, Kessler M, et al. Preventing 30-day hospital readmissions: a systematic review and meta-analysis of randomized trials. JAMA Intern Med. 2014;174(7):1095-1107.

19. Moore C, Wisnivesky J, Williams S, McGinn T. Medical errors related to discontinuity of care from an inpatient to an outpatient setting.J Gen Intern Med. 2003;18(8):646-651.

20. Adams MJD, Umbach PD. Nonresponse and Online Student Evaluations of Teaching: Understanding the Influence of Salience, Fatigue, and Academic Environments. Research in Higher Education. 2012;53(5):576591.

21. Ancker JS, Edwards A, Nosal S, et al. Effects of workload, work complexity, and repeated alerts on alert fatigue in a clinical decision support system. BMC Medical Informatics and Decision Making.2017;17(1):36.

22. Baseman JG, Revere D, Painter I, Toyoji M, Thiede H, Duchin J. Public health communications and alert fatigue. BMC Health Services Research. 2013;13(1):295.

Acknowledgements : The authors thank the Internal Medicine Residency Program at Mayo Clinic Florida, the Office of Quality Improvement Scholarship at Mayo Clinic, Dr. Colt Cowdell, and Dr. John Matulis for the support of this quality improvement initiative.

Conflict of Interest Statement : Each of the authors confirms that this manuscript has not been previously published and is not currently under consideration by any other journal. Each named author has substantially contributed to conducting the underlying research and drafting this manuscript. Additionally, all of the named authors have no conflict of interest, financial or otherwise.

Figures:

Figure 1. Initial resident survey. 


\section{Resident Discharge Planning Satisfaction Survey}

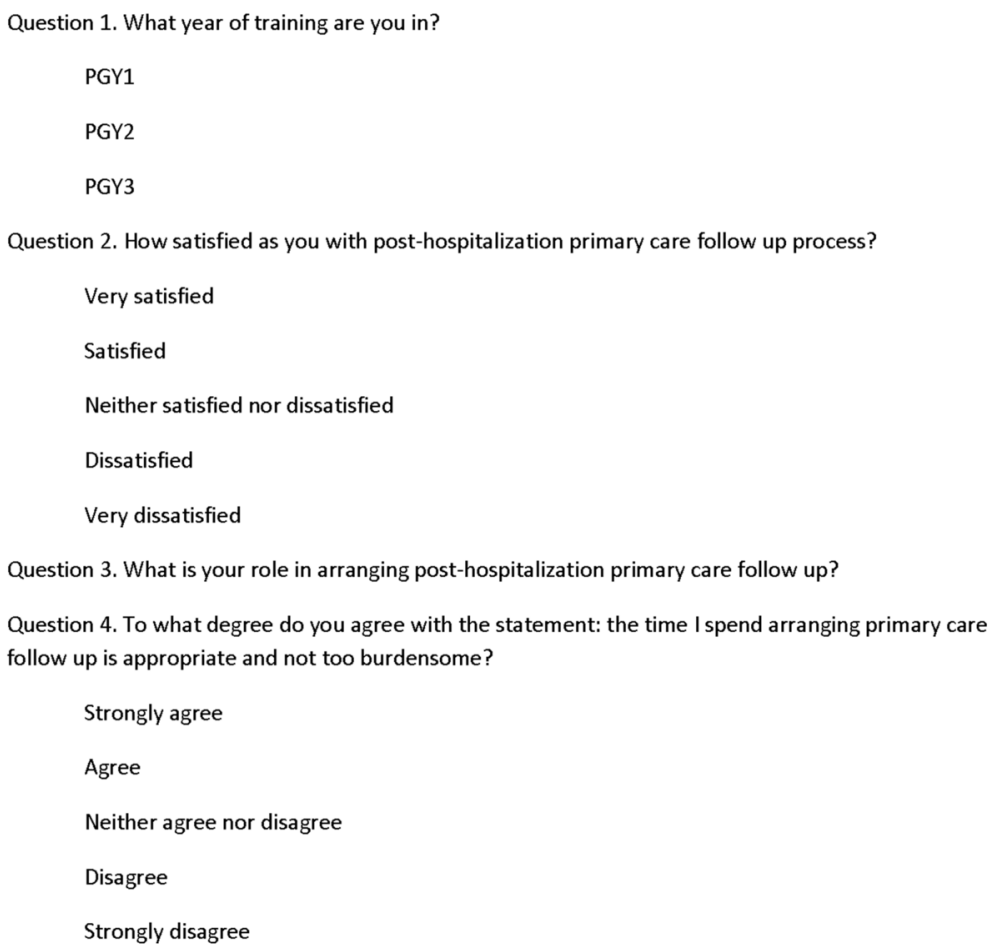

Figure 2. Fishbone diagram identifying factors leading to dissatisfaction amongst residents in the discharge planning process. 


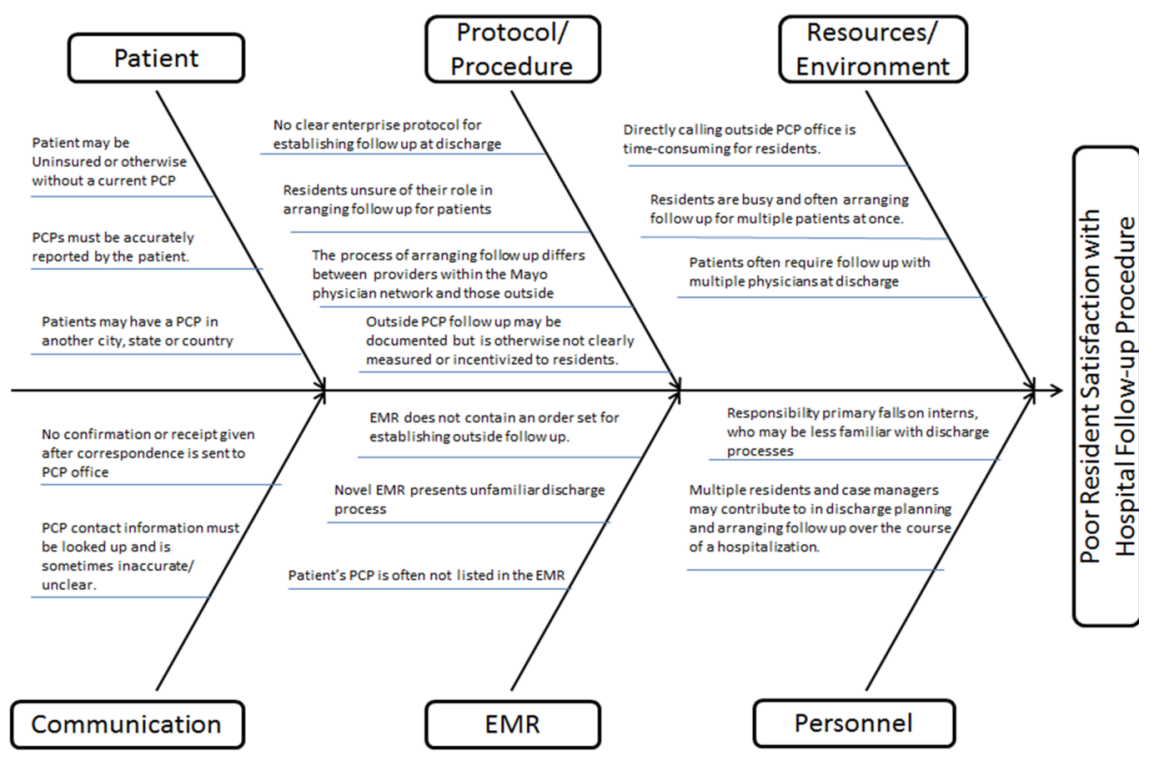

Figure 3. Flow diagram outlining initial process of establishing PCP follow-up at the time of discharge.

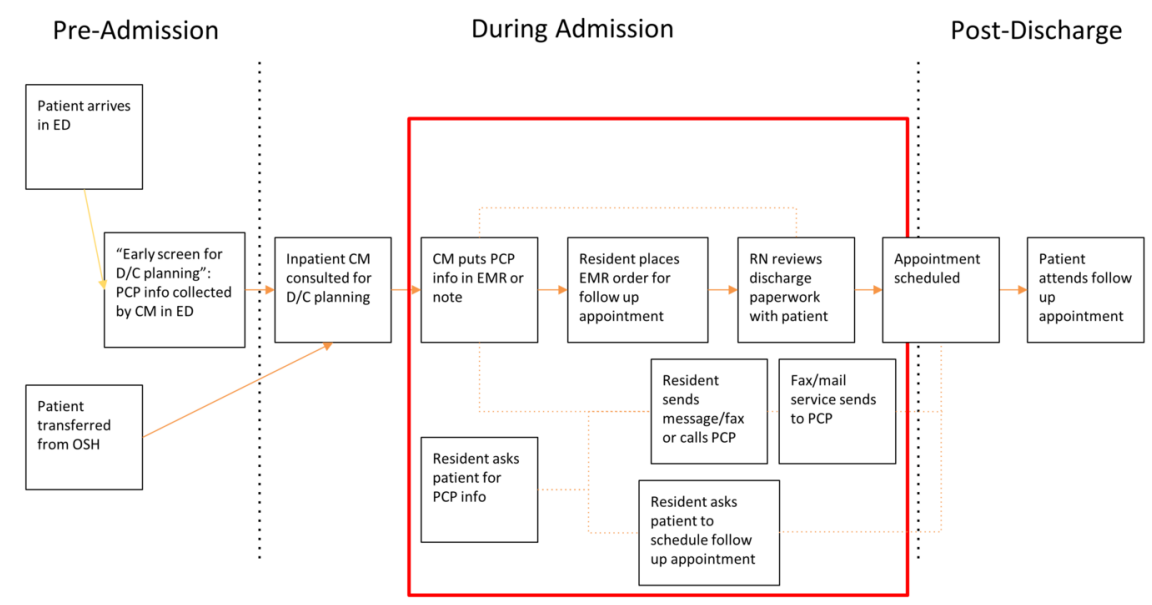

Figure 4. Pocket card outlining the streamlined process, using the current electronic medical record system, to effectively communicate with the patient's PCP and establish follow-up appointments and tests at the time of hospital discharge 
How To: Arrange For PCP Follow Up After Discharge

1. Find PCP information

a. Mayo PCP banner at top of chart, or

b. Click PCP banner and check under "Patient Care Team", or

i. Add "External PCP" info if not already entered

c. Written in case manager note

2. Discharge Order Set Instructions

a. Request 1 week (or sooner if applicable)

b. Mayo PCP

i. "Referrals and follow-Ups" tab

1. "Post Hospital Visit Primary Care"

ii. Or, create new order outside of $d / c$ order set

c. Outside PCP

i. "Discharge" tab

1. "F/U with non-Mayo PCP" $+/$-comments

2. "Bring a copy of these $d / c$ instructions to your follow up"

3. +/- "Other Discharge Instructions," e.g. labs/imaging to

repeat

3. Ordering Follow Up Labs/Tests

a. Mayo Labs

b. Outside Labs

i. Add order through $\mathrm{d} / \mathrm{c}$ order set, or as separate order

i. New order: "Outside Lab Tests - Req Only"

1. Will be printed to be sent home with patient

4. Verify Information in AVS Preview

a. Discharge Tab $\rightarrow$ After Visit Summary

b. Review appointments and other instructions

5. Post-Discharge Communication (after discharge summary complete)

a. Mayo PCP

i. "Route" discharge summary to PCP

b. Outside PCP

i. "Communication" Tab

1. To: "1 PCP", or "4 Care Team", or "+Add" to search for provider

a. Click contact info to select "fax" or "letter" correspondence

2. Letter Type: "Blank" or "Other Provider"

a. +/-edit letter, add any additional info/instructions for PCP

3. Attachments

a. "Create Document" $\rightarrow$ "Notes" $\rightarrow$ select d/c summary

b. +/- "Attach Report" for labs/imaging reports/etc

4. +/- "clean up letter" or "recipient info incomplete"

5. "Route Now"

ii. To verify when letter is sent, review under "Chart Review" $\rightarrow$ "Letters" tab

Figure 5. Resident satisfaction with the post-hospitalization primary care follow-up process across all three survey time points. 
How Satisfied are you with the post-hospitalization primary care follow up process?

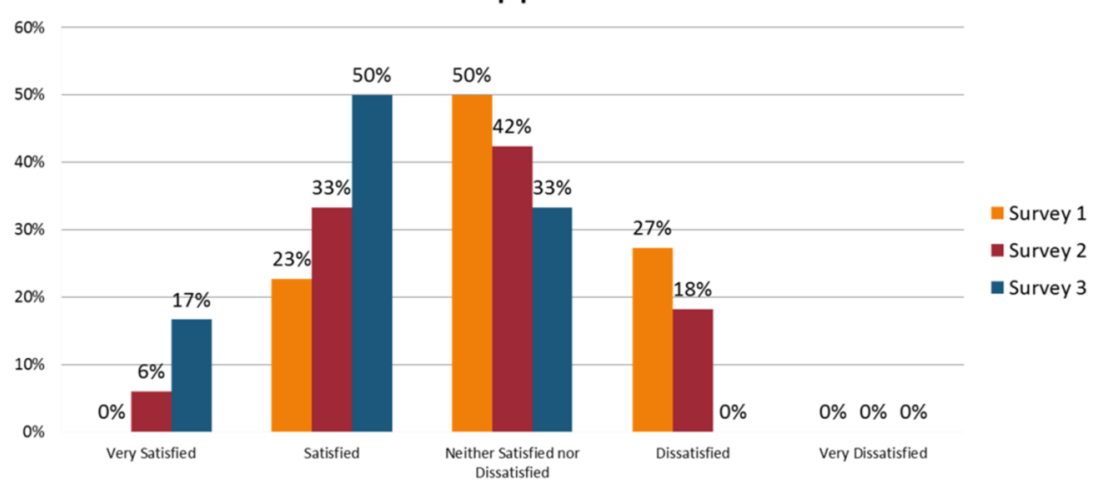

Figure 6. Resident perception of workload burden in establishing primary care follow-up across all three survey time points.






\section{Resident Discharge Planning Satisfaction Survey}

Question 1. What year of training are you in?

PGY1

PGY2

PGY3

Question 2. How satisfied as you with post-hospitalization primary care follow up process?

Very satisfied

Satisfied

Neither satisfied nor dissatisfied

Dissatisfied

Very dissatisfied

Question 3. What is your role in arranging post-hospitalization primary care follow up?

Question 4. To what degree do you agree with the statement: the time I spend arranging primary care follow up is appropriate and not too burdensome?

Strongly agree

Agree

Neither agree nor disagree

Disagree

Strongly disagree

Question 5. What aspects of the post-hospitalization primary care follow up process work well?

Question 6. What aspects of the post-hospitalization primary care follow up process don't work well?

Question 7. What do you think would improve how arrangements are made for PCP follow up? 


How To: Arrange For PCP Follow Up After Discharge

1. Find $P C P$ information

a. Mayo PCP banner at top of chart, or

b. Click PCP banner and check under "Patient Care Team", or

i. Add "External PCP" info if not already entered

c. Written in case manager note

2. Discharge Order Set Instructions

a. Request 1 week (or sooner if applicable)

b. Mayo PCP

i. "Referrals and Follow-Ups" tab

1. "Post Hospital Visit Primary Care"

ii. Or, create new order outside of $d / c$ order set

c. Outside PCP

i. "Discharge" tab

1. "F/U with non-Mayo $P C P$ " +/-comments

2. "Bring a copy of these $d / c$ instructions to your follow up"

3. +/- "Other Discharge Instructions," e.g. labs/imaging to

repeat

3. Ordering Follow Up Labs/Tests

a. Mayo Labs

i. Add order through $\mathrm{d} / \mathrm{c}$ order set, or as separate order

b. Outside Labs

i. New order: "Outside Lab Tests - Req Only"

1. Will be printed to be sent home with patient

4. Verify Information in AVS Preview

a. Discharge Tab $\rightarrow$ After Visit Summary

b. Review appointments and other instructions

5. Post-Discharge Communication (after discharge summary complete)

a. Mayo PCP

i. "Route" discharge summary to PCP

b. Outside PCP

i. "Communication" Tab

1. To: "1 PCP", or "4 Care Team", or "+Add" to search for provider

a. Click contact info to select "fax" or "letter" correspondence

2. Letter Type: "Blank" or "Other Provider"

a. +/-edit letter, add any additional info/instructions for PCP

3. Attachments

a. "Create Document" $\rightarrow$ "Notes" $\rightarrow$ select d/c summary

b. +/- "Attach Report" for labs/imaging reports/etc

4. +/- "clean up letter" or "recipient info incomplete"

5. "Route Now"

ii. To verify when letter is sent, review under "Chart Review" $\rightarrow$ "Letters" tab 
How Satisfied are you with the post-hospitalization primary care follow up process?

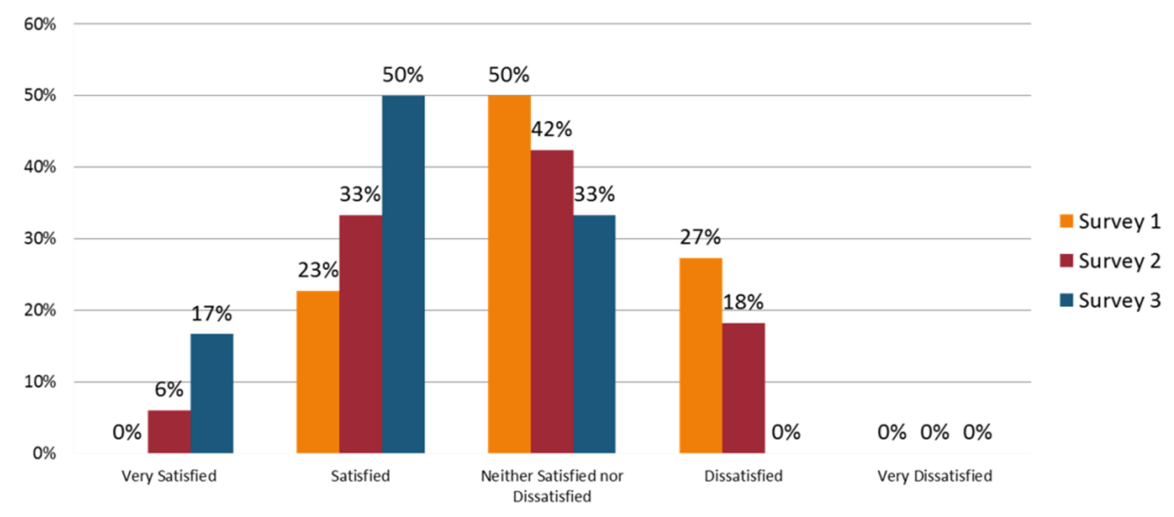

The time I spend arranging primary care follow up is appropriate and not too burdensome

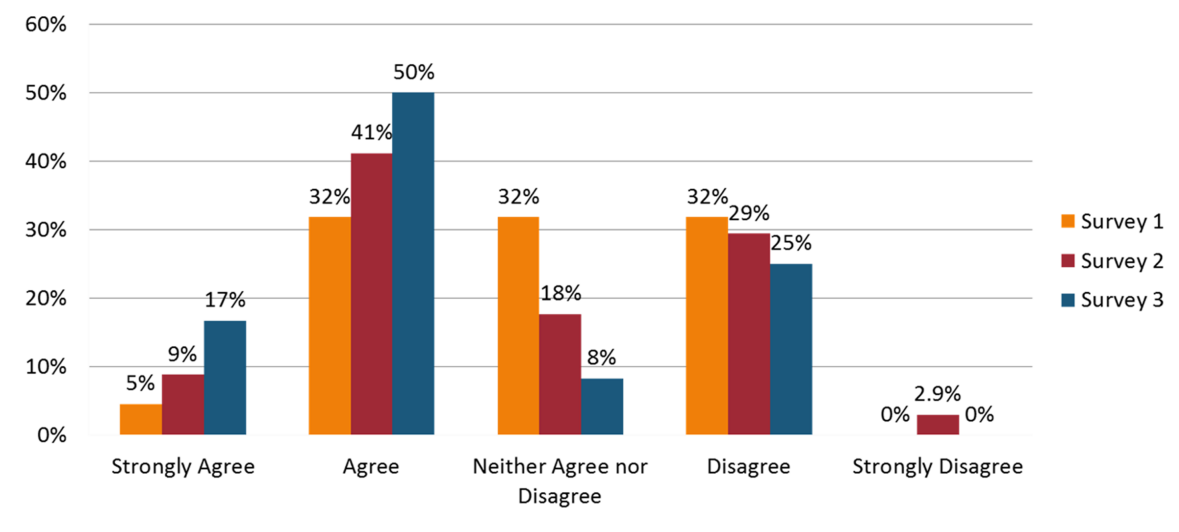

